\title{
Fecal Microbiota Transplantation for the Treatment of Nonalcoholic Fatty Liver Disease
}

\author{
Lan-Feng Xue, Wen-Hui Luo, Li-Hao Wu, Xing-Xiang He, Harry Hua-Xiang Xia and Yu Chen* \\ Department of Gastroenterology, The First Affiliated Hospital of Guangdong Pharmaceutical University, Guangzhou, \\ Guangdong Province, China
}

\begin{abstract}
Nonalcoholic fatty liver disease (NAFLD) is significantly on the rise, which will seriously affect human health; yet, there is no approved drug for the treatment of NAFLD currently. Recently, more and more studies have demonstrated that intestinal bacteria affect liver function through the gut-liver axis, and that the imbalance of intestinal bacterial composition is essential in the pathogenesis of NAFLD. Correcting intestinal bacterial imbalance, therefore, may prevent the development and attenuate the progression of NAFLD. Fecal microbiota transplantation (FMT) is considered the most effective method to correct intestinal bacteria imbalance, but its therapeutic role in NAFLD has not been established. Moreover, the potential molecular mechanisms of FMT for NAFLD have not been elucidated. This review paper summarizes the currently available experimental and clinical research results on the therapeutic effects of FMT for NAFLD. In addition, the underlying molecular mechanisms are proposed, which would provide theoretical support for FMT as a useful modality for the treatment of NAFLD.
\end{abstract}

Introduction

Nonalcoholic fatty liver disease (NAFLD) is one of the currently most prevalent chronic liver diseases, being histologically categorized into nonalcoholic fatty liver (NAFL), nonalcoholic steatohepatitis (NASH), and nonalcoholic fatty liver cirrhosis. ${ }^{1}$ Previous studies have shown that one fourth of the worldwide population have NAFLD, as diagnosed by imaging, with the incidence rate ranging from 19.9 to 29.7 per 1,000 person-years and the prevalence ranging from $13.5 \%$ to $31.8 \% .^{1}$ Following the westernization of diet and lifestyle over the past decades, the prevalence of NAFLD in the Chinese population has been increasing, from $18.2 \%$ in 2000-2006 to $20.0 \%$ in $2007-2009$ and $20.9 \%$ in $2010-2013 .^{2}$

Following long-term complex interactions among the intestinal bacteria, diet, heredity, obesity and the other various factors, NAFLD, especially NASH, can evolve from a pure fatty liver to

Keywords: Non-alcoholic fatty liver disease; Intestinal bacteria; Fecal microbiota transplantation; Gut-liver axis; Molecular mechanisms.

Abbreviations: DAMP, damage-associated molecular patterns; FAP, fat attenuation parameter; FMT, fecal microbiota transplantation; HFD, high-fat diet; LPS, lipopolysaccharides; NAFLD, nonalcoholic fatty liver disease; NASH, nonalcoholic steatohepatitis; PAMP, pathogen-associated molecular pattern.

Received: December 23, 2018; Revised: February 28, 2019; Accepted: March 05, 2019

${ }^{*}$ Correspondence to: Yu Chen, Department of Gastroenterology, the First Affiliated Hospital of Guangdong Pharmaceutical University, Guangzhou 510080, Guangdong Province, China. Tel: +8613824462875; E-mail: yuchen@gdpu.edu.cn

How to cite this article: Xue L-F, Luo W-H, Wu L-H, He X-X, Xia HH-X, Chen Y. Fecal Microbiota Transplantation for the Treatment of Nonalcoholic Fatty Liver Disease. Exploratory Research and Hypothesis in Medicine 2019;4(1):12-18. doi: 10.14218/ERHM.2018.00025. liver fibrosis, to cirrhosis and even to hepatic cellular carcinoma. ${ }^{3}$ It is estimated that NAFLD would become the most frequent indication for liver transplantation by $2030 .{ }^{4} \mathrm{Up}$ to now, there is no effective drug to treat NAFLD. Thus, lifestyle modification, ${ }^{5}$ including physical exercise, healthy diet and weight loss, is generally recommended as the primary clinical management to alleviate NAFLD, or to slow down or even cease the progression to fibrosis, cirrhosis or even carcinoma. ${ }^{2}$ However, the expected therapeutic efficacy cannot be achieved in a substantial proportion of patients due to poor patient compliance, and few patients can achieve adequate weight reduction and even fewer can maintain the weight in the long run..$^{5}$ Therefore, searching for novel effective therapeutic strategies is an urgent medical issue for the management of NAFLD.

In recent years, an increasing number of studies have shown that alteration of gut microbiota, or the imbalance of intestinal bacteria, plays an essential role in the pathogenesis of NAFLD. ${ }^{6-9}$ The pathogenic pathways leading to liver damage include activation of pathogen-associated molecular patterns (PAMPs) and damageassociated molecular patterns (DAMPs). When intestinal microecology imbalance causes intestinal dysfunction, these pathways can be activated and lead to liver disease. Therefore, actively correcting the altered gut microbiota is believed to be a new therapeutic approach for NAFLD.

Currently, a variety of commercial oral probiotic supplements, in the form of yogurt or pill, have been applied to ameliorate the dysbiosis. ${ }^{10,11}$ Unfortunately, their clinical efficacies for NAFLD is limited or remain to be confirmed; this is mainly due to the fact that the supplements used are single species, low amount, and low vitalityIn addition, larger randomized studies are still lacking, as stated in a Cochrane meta-analysis. ${ }^{12}$ Fecal microbiota transplanta- 
tion (FMT), also known as fecal bacteria transplantation, is a recently developed novel therapeutic option for several diseases. ${ }^{13-16}$ Compared with oral probiotics, FMT can significantly improve the disorder of intestinal bacteria, and is currently recognized as the most valid method to restore the intestinal micro-ecological balance. ${ }^{17}$ The therapeutic role of FMT in NAFLD has been evaluated in both animal and clinical studies, and accumulating evidence indicates that FMT is promising in treating NAFLD. However, the underlying molecular mechanisms are not entirely understood, and well-designed, large-scale randomized clinical trials are needed to confirm the efficacy.

In the present review, we summarize the existing experimental and clinical evidence of FMT exerting therapeutic effects on NAFLD and hypothesize that FMT exerts the therapeutic effects by blocking the above-mentioned four pathways.

\section{FMT concept and history}

FMT, also known as a stool transplant, is a bacteriotherapy, which is the process of transplantation of fecal bacteria from a healthy individual into a recipient. ${ }^{18}$ FMT is an effective strategy to restore intestinal microbial diversity. ${ }^{19}$ The earliest history of fecal bacteria infusion in medicine can be traced back to ancient China in the 4th century. ${ }^{16}$ In ancient Chinese medical literature, the term "Huang Tang (yellow soup)" was used to successfully cure cases of severe food poisoning and diarrhea. However, the treatment role of fecal bacteria did not receive much approval in western countries, and was even considered as a disgusting "folk prescription" until the $21^{\text {st }}$ century.

In 2013, FMT was written into the clinical guidelines for the treatment of refractory and recurrent Clostridium difficile infection in the USA, ${ }^{20}$ indicating the leapfrog status of FMT in the history of medicine. Subsequently, FMT has become a hot research field worldwide. FMT is currently considered a new efficacious modality for treating immune and metabolic diseases, such as diabetes mellitus, obesity, and NAFLD. One of the advantages is that the bacteria used for transplantation are viable. The other advantage is that the amount of transplanted bacteria is tremendous. Various studies have demonstrated that the imbalance of intestinal microecological environment can result in or aggravate hepatocyte steatosis and hepatitis. ${ }^{21,22}$ Therefore, FMT can transform the composition of the gut bacteria spectrum, increase the richness of healthy intestinal bacteria, inhibit intestinal bacterial overgrowth and repair intestinal barrier permeability, which subsequently regains the dominant position of healthy intestinal bacteria. ${ }^{13,16}$

\section{Anatomical structure and physiological function: gut-liver axis}

The gut-liver axis is an essential bridge between the intestine and liver, representing the major pathway through which intestinal bacteria exert their effects on the liver function. Anatomically, $70 \%$ of the blood supply of the liver comes from the portal vein, and the liver contacts with the intestine mainly by the portal vein system, which makes liver vulnerable to the damage of intestinal bacteria and its endotoxins. ${ }^{23}$ Physiologically, on one hand, the metabolites, such as substances broken-down from food, and the secondary bile acids, and enterobacteria in the intestine, can be absorbed into the intestinal vein, flow into the portal vein, and then reach the liver tissue. ${ }^{24}$ On the other hand, substances such as the primary bile acid, IgA, and antibacterial molecules produced by the liver, can be secreted into the biliary tract, and arrive in the intestine. Ad- ditionally, inflammatory mediators (such as IL-1 $\beta$, trimethylamine oxide, etc.) and intestinal metabolites (such as acetaldehyde, butyric acid, ethanol, etc.) generated by metabolism in the liver can all infiltrate into the systemic circulation and keep two-way communication.

It is the presence of these anatomical structures and physiological functions that form the gut-liver axis, through which the liver and the intestine functionally interact with or influence each other. When the intestinal flora is disturbed, toxic substances produced by the harmful bacteria enter into the liver through the above pathways, leading to the damage of the liver function. FMT can weaken harmful bacteria in the intestine, decrease the quantity of toxins entering the gut-liver axis, and thus prevent further damage to the liver. Therefore, the gut-liver axis is the critical structure of FMT to treat with NAFLD (Fig. 1).

\section{Experimental evidence}

There are several animal experiments that have explored the potential beneficial therapeutic effects of FMT for NAFLD (Table 125-28).

In a study conducted by Le Roy et al., ${ }^{25}$ two male C57BL/6J mice, representing high-fat diet (HFD) "responder" and "nonresponder", respectively, were selected as donors. Both mice had similar body weight. Whereas the "responder" developed hyperglycemia with a high plasma concentration of pro-inflammatory cytokines, the "nonresponder" was normoglycemic with a lower level of systemic inflammation. Then, 8-week-old germ-free mice were transplanted with intestinal microbiota from either the "responder" $(n=22)$ or the "nonresponder" $(n=18)$ through intragastric perfusion, and fed the same HFD for 16 weeks. It was found that although all mice developed comparable obesity, fasting hyperglycemia and insulinemia developed in the responder-receivers but not in the nonresponder-receivers. In addition, hepatic macrovesicular steatosis developed in the responder-receivers but not in the nonresponder-receivers. Moreover, the intestinal bacteria were different at the phylum, genera and species levels between the two groups, as determined by $16 \mathrm{~S}$ rRNA sequencing.

Zhou et al. $^{26}$ randomly divided specific pathogen-free male C57BL/6 mice into control, HFD, and HFD+FMT groups $(n=12$ in each group). Whereas the control group was fed with a standard diet, and the HFD group was fed HFD for 16 weeks, the HFD+FMT group was fed HFD for 16 weeks and received FMT for the latter 8 weeks. FMT was carried out by transplanting the feces collected from the control group into the intestine by gavage. They found that compared with the control group, intestinal permeability and hepatic steatosis were increased in the HFD group. However, compared with the HFD group, the intestinal permeability and hepatic steatosis were significantly reduced in the HFD+FMT group. These findings indicate that FMT could alleviate HFD-induced steatohepatitis in mice via beneficial regulation of gut microbiota.

Soderborg et al. ${ }^{27}$ divided pregnant women into obesity ( $n=$ 8 ) and normal weight $(n=7)$ groups, and then collected and separately pooled the feces from their 2 -week-old infants to feed two groups of GF C57BL/6J mice for 21 days ( $n=12$ per group). They found that the GF mice colonized with fecal microbes from infants born to obese mothers showed increasing intestinal permeability, hepatic gene expression for endoplasmic reticulum stress and innate immunity, together with histological signs of periportal inflammation and excess weight, as compared with those colonized with fecal microbes from infants born to obese mothers from normal-weight mothers. These findings provide experimental evidence that maternal obesity-associated infant dysbiosis may play a 


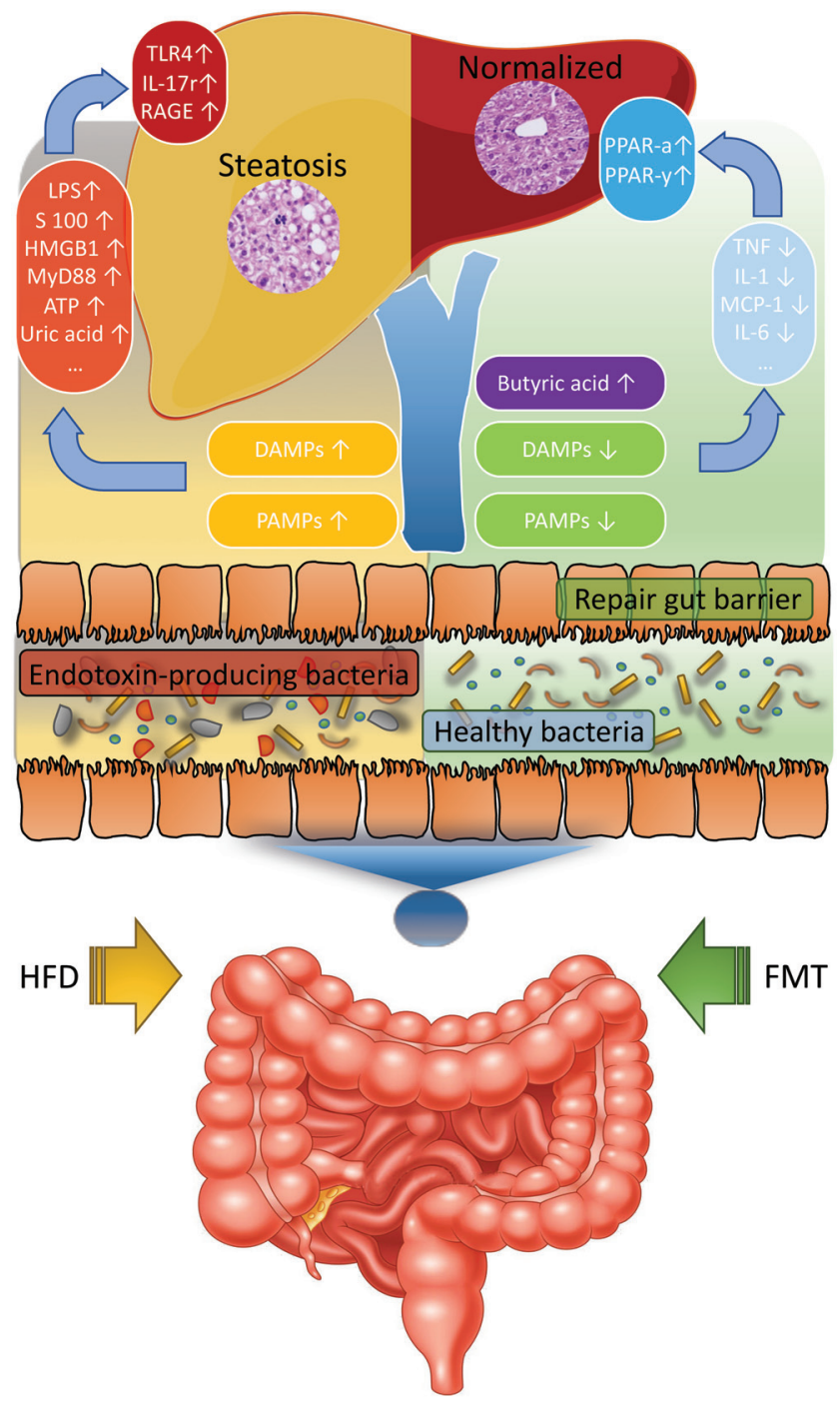

Fig. 1. Proposed molecular mechanism of FMT for the treatment of NAFLD. The increase of endotoxin-producing bacteria in the intestine leads to intestinal mucosal injury. Endotoxin and other harmful substances activate inflammatory pathways, such as DAMPs and PAMPs, and further produce a series of inflammatory factors, which promote NAFLD through the liver-gut axis (shown in the left side of the figure). After FMT, healthy bacteria would be increased, which repairs intestinal mucosal barrier, inhibits pathogenic pathways, such as DAMPs and PAMPs, and promotes anti-inflammatory factors to improve NAFLD (shown in the right side of the figure). Abbreviations: ATP, adenosine triphosphate; DAMPs, damageassociated molecular patterns; FMT, fecal microbiota transplantation; HFD, high-fat diet; HMGB1, high mobility group box 1; MyD88, myeloid differentiation factor 88; NAFLD, nonalcoholic fatty liver disease; PAMPs, pathogen-associated molecular patterns; PPAR, peroxisome proliferatorsactivated receptors; RAGE, receptor of advanced glycation end products; TLR4, toll-like receptor 4 .

causative role in childhood obesity and NAFLD.

García-Lezana et al. ${ }^{28}$ fed Sprague-Dawley rats with a high-fat, high-glucose/fructose diet or a control diet/water for 8 weeks, and then selected representative rats as the intestinal microbiota donors. Then, they transplanted the feces of the representative rats into additional rats fed with either the high-fat, high-glucose/fructose diet or control diet/water, and maintained the diet for 12-14 days. They observed that the rats fed high-fat, high-glucose/fructose diet developed obesity, insulin resistance, NASH without fibrosis, portal hypertension, intrahepatic endothelial dysfunction, and intestinal microbiota dysbiosis. Transplantation with feces from control diet/ water donors significantly reduced portal hypertension, although there was no significant change in NASH. In addition, the weight of the rats was significantly decreased, and the insulin resistance was improved. These findings indicate that FMT is potentially a therapeutic option for portal hypertension in NASH.

Therefore, these experimental findings indicate that intestinal bacteria play an important role in body metabolism, and intestinal bacterial disorder could lead to various metabolic diseases, such as fatty liver, obesity, insulin resistance, etc. FMT, which transplants the healthy intestinal bacteria into the gut of animals with metabolic diseases, can rebalance the intestinal bacteria in situ to improve the metabolic symptoms, and even reduce the fat deposition in the liver.

\section{Clinical research}

Up to now, no clinical data have been reported on the efficacy of FMT treatment for NAFLD. Here, we present preliminary data of a pilot prospective study (ChiCTR1800018920, http://www.chictr. org.cn/edit.aspx?pid=31990\&htm=4) currently being carried out in our department. The study, which was performed with the approval of the Ethical Committees of the First Affiliated Hospital of Guangdong Pharmaceutical University, aimed to determine the effect of FMT on hepatic steatosis in patients with NASH. In this prospective study, patients with NAFLD were divided into two groups, which were the FMT group and the non-FMT control group. A total of 47 patients with NAFLD were recruited between April 1, 2017 and September 30, 2018: 27 received FMT ( $200 \mathrm{~mL} / \mathrm{d}$ for 3 days; FMT group) and 20 who did not receive special treatment for NAFLD served as controls (non-FMT group). Data on blood biochemistry and FibroTouch, which was used to determine fat attenuation parameter (FAP), ${ }^{29}$ were recorded from the 27 patients before and 1 month after FMT treatment, as well as for the 20 controls. The average values of FAP before and after treatment are described in Table 2. It was found that the difference in the change of FAP as measured by FibroTouch was significant after treatment with FMT, compared with the control group ( $\mathrm{F}=$ $4.603, p=0.038$, by analysis of covariance) (Table 2 ). The values of change in FAP are $-9.26 \pm 29.31$ in the FMT group and $12.21 \pm$ 24.48 in the control group. This finding indicates that FMT indeed attenuates hepatic steatosis.

Therefore, we conclude, from our preliminary and first ever reported data, that FMT improves the fatty liver disease, and the completion of our present study would further consolidate the conclusion. According to clinicaltrials.gov, one clinical trial that evaluates if FMT can alter insulin resistance and reduce the amount of fat deposited in the liver in patients with metabolic syndrome and NAFLD has been recently completed in Canada (NCT02496390). No results have been reported yet. In addition, two more clinical trials that assess the efficacy and safety of FMT for NASH (NCT02469272) and NASH-related cirrhosis (NCT02721264) are currently recruiting subjects.

Proposed hypothesis on the molecular mechanisms of FMT for the treatment of NAFLD

As mentioned above, FMT is a potential modality for the treatment of NAFLD. ${ }^{13,16,30,31}$ However, the underlying molecular 
Table 1. Animal experiments on FMT in the treatment of nonalcoholic fatty liver disease

\begin{tabular}{|c|c|c|c|c|}
\hline Author/publication year & Animal models & Control & Intervention & Results \\
\hline Le Roy et al./2013 25 & $\begin{array}{l}\text { One } \text { C57BL/6J } \\
\text { mice (HFD } \\
\text { "responder") }\end{array}$ & $\begin{array}{l}\text { One C57BL/6J } \\
\text { mice (HFD } \\
\text { "nonresponder") }\end{array}$ & $\begin{array}{l}\text { The microbiota } \\
\text { of "responder" or } \\
\text { "nonresponder" transplanted } \\
\text { to GF mice }(\mathrm{RR}=22, \mathrm{NRR}=18)\end{array}$ & $\begin{array}{l}\text { RR: developed fasting } \\
\text { hyperglycemia and insulinemia, } \\
\text { hepatic steatosis } \\
\text { NRR: Normal blood glucose }\end{array}$ \\
\hline Zhou et al./2017²6 & $\begin{array}{l}\text { SPF male } \\
\text { C57BL/6 mice: } \\
\operatorname{HFD~}(n=12) \\
\operatorname{HFD}+\mathrm{FMT}(n=12)\end{array}$ & $\begin{array}{l}\text { SPF male } \\
\text { C57BL/6 mice: } \\
\text { Normal diet } \\
(n=12)\end{array}$ & $\begin{array}{l}\text { SPF male C57BL/ } 6 \text { mice } \\
\text { divided into three groups } \\
\text { and fed with a normal diet or } \\
\text { high fat diet respectively for } \\
16 \text { weeks. "HFD+FMT" group } \\
\text { has additional therapy of } \\
\text { fecal microbiota for } 8 \text { weeks. }\end{array}$ & $\begin{array}{l}\text { HFD induced liver steatohepatitis } \\
\text { of mice, and FMT attenuated } \\
\text { steatohepatitis in mice. }\end{array}$ \\
\hline Soderborg et al./2018 27 & $\begin{array}{l}\text { GF mice } \\
\text { colonized with } \\
\text { the microbiota of } \\
\text { infant of obesity } \\
\text { mother }(n=12)\end{array}$ & $\begin{array}{l}\text { GF mice } \\
\text { colonized with } \\
\text { the microbiota } \\
\text { of infant of } \\
\text { normal weight } \\
\text { mother }(n=12)\end{array}$ & $\begin{array}{l}\text { Randomly selecting four mice } \\
\text { from each group, exposure to } \\
\text { a WSD. Repeat three times. }\end{array}$ & $\begin{array}{l}\text { GF mice colonized with the } \\
\text { microbiota of infant of obesity } \\
\text { mother: intestinal permeability } \uparrow \\
\text { hepatic gene expression } \\
\text { for endoplasmic reticulum } \\
\text { stress } \uparrow \text { excess weight gain }\end{array}$ \\
\hline $\begin{array}{l}\text { García-Lezana } \\
\text { et al./2018 }\end{array}$ & $\begin{array}{l}\text { Male Sprague- } \\
\text { Dawley rats: } \\
\text { HFGFD }(n=6)\end{array}$ & $\begin{array}{l}\text { Male Sprague- } \\
\text { Dawley rats: } \\
\text { Control diet } \\
(n=6)\end{array}$ & $\begin{array}{l}\text { HFGFD and CD rats were } \\
\text { transplanted with microbiota } \\
\text { from opposite diet donors: } \\
\text { heterologous transplant or } \\
\text { autologous fecal transplant. }\end{array}$ & $\begin{array}{l}\text { HFGFD rats developed obesity, } \\
\text { insulin resistance, NASH with PH, } \\
\text { and the HFGFD autotransplanted } \\
\text { rats were decrease PH. }\end{array}$ \\
\hline
\end{tabular}

Abbreviations: CD, control diet/water; FMT, fecal microbiota transplant; GF, germ-free; HFD, high fat diet; HFGFD, high-fat, high-glucose/fructose diet; NRR, nonresponderreceivers; RR, responder-receivers (the responder developed obesity, fasting hyperglycemia and insulinemia after HFD; SPF, specific pathogen-free; WSD, Western-style diet.

mechanisms are still in the exploratory stage and have not been fully clarified. It is known that activation of PAMPs and DAMPs are the main pathogenic pathways leading to liver damage, as described earlier. Based on the existing experimental findings, we would summarize and put forward the hypothesis on the molecular mechanisms by targeting these two pathways (Fig. 1), which are described in detail below.

Reduce the inflammatory response by transforming the composition of intestinal bacteria, reduce endotoxin-producing bacteria and increase probiotics

The intestinal bacteria of NAFLD patients often present the characteristics of the increased distribution of Gram-negative bacteria. Lipopolysaccharides (LPS), also known as lipoglycans and endotoxins, are the main component of the cell wall of Gram-negative bacteria. They are the primary pathogenic substances that can increase the permeability of the intestinal barrier and activate the intestinal immune cells' secretion of inflammatory mediators, leading to the occurrence of autoimmune intestinal inflammation. Meanwhile, LPS can be transported into the liver through the gutliver axis, combine with the receptors (e.g., TLR4, IL-17r) on the immune cell surface in the liver, activate immune cells to secrete inflammatory factors, such as TNF- $\alpha$, IL- 6 and IL- $1 \beta$, and further initiate a hepatic inflammatory response. ${ }^{32}$ Transplantation of the integral fecal bacteria derived from healthy people to overturn the composition of intestinal bacteria in patients would result in reduction of the endotoxin production and endotoxin-mediated inflammatory reaction, and maintain the ecological balance of intestinal bacteria.

Besides, FMT can increase the proportion of probiotics, such as Lactobacilli. These probiotics produce some critical metabolites, such as butyrate by fermentation, and participate in the division and decomposition of substances to promote the metabolic balance of the body. Butyrate, which is a short chain fatty acid, is demonstrated to be an immunomodulator and anti-inflammatory substance, and is involved in protecting the intestinal mucosa barrier, regulating immune function and increasing insulin receptor sensitivity. ${ }^{33-37}$ Zhang et al. ${ }^{38}$ found that the intestinal inflammatory response in the animal model was significantly improved after transplanting butyrate bacteria extracted from healthy people into the animals with HFD-induced NAFLD, suggesting that butyrate has an anti-inflammatory effect. In addition, butyrate has also been found to be a fundamental substance for maintaining the intestinal barrier and immune regulation. ${ }^{34,39}$ Moreover, probiotics may

Table 2. Effect of FMT on fat attenuation in patients with nonalcoholic fatty liver disease

\begin{tabular}{llllll}
\hline Variable & Stage & FMT $(\boldsymbol{n}=\mathbf{2 7})$, Mean \pm SD & Controls $(\boldsymbol{n}=\mathbf{2 0})$, Mean \pm SD & F & \\
\hline FAP value & Before & $279.37 \pm 34.14$ & $244.70 \pm 41.36$ & \\
& After & $270.11 \pm 44.04$ & $256.91 \pm 45.56$ & \\
Change in FAP & After-before & $-9.26 \pm 29.31$ & $12.21 \pm 24.48$ & 4.603 & 0.038 \\
\hline
\end{tabular}

The data are derived from a prospective clinical study currently being conducted at the First Affiliated Hospital of Guangdong Pharmaceutical University, Guangzhou, China. Abbreviations: SD, standard deviation. FAP, fat attenuation parameter; FMT, fecal microbiota transplantation. Comparison was between the FMT group and the non-FMT group. 
also produce many other metabolic substances to prevent the body from damage. Therefore, FMT can transform the composition of intestinal flora, increase the number of probiotics and their beneficial metabolites to achieve the goals of improving the intestinal barrier function, decreasing the pathogenic factors and reducing the inflammatory response in the liver.

\section{Repair the intestinal mucosa mechanical and immune barriers by increasing the production of tight junction proteins}

The intestinal mucosa barrier can effectively stop harmful substances (bacteria or toxins) from entering into tissues and the blood circulation. If the tight junction between the intestinal epithelial cells is destroyed, the intestinal permeability will increase and result in LPS penetrating the submucosal blood vessel, ultimately causing a liver lesion. In an animal study, Zhou et al. ${ }^{26}$ transplanted the feces taken from normal mice into mice with HFD-induced NAFLD. After 8 weeks of the treatment, the beneficial bacteria and the levels of intestinal tight binding protein ZO-1 and butyrate were increased in the intestine of NAFLD mice, and the serum level of LPS was decreased. In addition, the accumulation of lipid and inflammatory factors in liver were significantly reduced after FMT. These findings suggest that FMT could reduce the intestinal permeability and repair mechanical barriers in the gut by increasing the expression of tight junction proteins.

Additionally, the intestinal mucosal immune barrier may also be enhanced by increased butyrate production after FMT, which increases the production of butyrate-producing bacteria. An animal study by Smith et al. ${ }^{36}$ showed that the number of Treg cells was increased and the anti-inflammatory factor IL-10 was activated in the intestine of sterile mice fed with sodium butyrate. Other studies have shown that butyrate also reduces release of pro-inflammatory cytokines, inhibiting the intestinal inflammatory response and maintaining the intestinal immune balance, probably by modulating the activity of related inflammatory pathways, such as G-protein coupled receptor, NF- $\kappa \mathrm{B}$, and JAK/STAT. ${ }^{37,40}$ Therefore, FMT would be the therapeutic approach for NAFLD by enhancing the intestinal mechanical barrier and immune barrier.

\section{Increase the expression of insulin receptors in the liver and im- prove insulin resistance by increasing butyrate production}

Insulin resistance is one of the mechanisms responsible for lipid metabolic disorder in the liver, by increasing lipid deposition, aggravating lipid degeneration and causing oxidative stress in the liver. Therefore, increasing the expression of the insulin receptor and the sensitivity of insulin can improve the lipid deposition of hepatocytes and reduce inflammation caused by lipid oxidative stress. Jin et al. ${ }^{35}$ showed that the expression of the insulin receptor in the liver was significantly upregulated, and insulin sensitivity and hepatic steatosis was significantly improved after administration of microbial butyrate into mice with NASH. These findings, in combination with the fact that FMT increases the proportion of intestinal butyrate-producing bacteria, as mentioned above, further indicate that FMT can increase the production of butyrate and subsequently promote the expression of the insulin receptor.

Furthermore, Zhou et al. ${ }^{26}$ also found that the intestinal butyrate was significantly increased after transplantation, which was associated with decreased serum levels of a series of inflammatory factors (e.g., TNF, IL-1, MCP-1, IL-6) and intrahepatic levels of triglyceride and cholesterol. The PPAR- $\alpha$ mRNAs, from a gene that mainly participates in fatty acid oxidation, in the liver were significantly upregulated in the HFD group compared with the control group and significantly downregulated after FMT intervention. The protein level of the insulin receptor was upregulated, following a significant increase of butyrate concentration in the cecal content after FMT. Therefore, FMT can improve the expression of the insulin receptor in the liver by increasing the production of butyrate bacteria, acting on the improvement of insulin resistance and reducing liver cell fat deposition to maintain lipid metabolic balance.

\section{Attenuate the activation and migration of DAMPs by downregu- lating their receptors}

DAMPs are host biomolecules that can initiate and perpetuate the primary mechanism of sterile inflammation and are released to the damaged site during cell or tissue injury; they include the high mobility group box 1 protein, S100 protein, nuclear DNA, etc. ${ }^{41}$ In NAFLD, DAMPs are secreted from the activated immune cells, whereas hepatic immune cells receive the signals of cell death or intestinal injury (e.g., LPS binding to receptors on the surface of liver immune cells), migrate to the position of inflammatory lesions, and then bind to their corresponding receptors to aggravate the intrahepatic inflammatory response. ${ }^{32}$ As long as the lesion signal is not relieved, its inflammatory effect is sustained, which is also one of the crucial links in the pathogenesis of NAFLD, DAMPs combine with the receptor of advanced glycation end products or toll-like receptor family members (including the TLR4 receptor), participate in the inflammatory response to the intestine and subsequently are involved in the development of NAFLD. ${ }^{42}$ FMT can both alleviate the production of LPS and the secretion of DAMPs. Moreover, FMT can downregulate the MyD88 protein of TLR4 receptors, inhibit the expression of TGF-1 and $\alpha$-SMA, and then reduce the number of receptors that can bind to DAMPs. ${ }^{26,42}$ In other words, FMT can reduce or block the continuous inflammatory infiltration in the liver by downregulating the receptors of DAMPs and subsequently attenuating their activation and migration.

\section{Prospects}

FMT is a medical breakthrough in recent years but it is currently still in the exploratory stage. The clinical efficacy of FMT for NAFLD needs to be further explored and confirmed in well-designed clinical studies. Moreover, the proposed molecular mechanisms are worth being investigated or elucidated. Although several critical issues, including quality of the donors, consumer acceptance, and ethical, technological and regulatory considerations, exist prior to widespread implementation and application in clinical practice, we are confident that these issues can be gradually resolved with the promising efficacy of FMT for the treatment of NAFLD.

\section{Conclusions}

FMT improves the physiological and biological parameters and symptoms of NAFLD, as shown in animal experiments and our preliminary clinical study. It is proposed that FMT may treat NAFLD by reducing the inflammatory response, repairing intestinal mechanical and immune barriers, upregulating insulin receptors and improving insulin resistance and attenuating the activation and migration of DAMPs. 


\section{Acknowledgments}

The authors would like to thank Quanzhen Chen, Zelin Wu, and Sihang Lin for their assistance in data collection. This work was supported by the Science and Technology Planning Project of Guangdong Province, China (2015A030302085 and 2016A020212022 to Y.C.).

\section{Conflict of interest}

The authors have no conflict of interests related to this publication.

\section{Author contributions}

Study design (XXH, YC); writing of the article (LFX); data acquisition, data analysis and interpretation (WHL, LHW); editing, reviewing, and final approval of the article (YC, HHX).

\section{References}

[1] Chalasani N, Younossi Z, Lavine JE, Charlton M, Cusi K, Rinella M, et $a l$. The diagnosis and management of nonalcoholic fatty liver disease: Practice guidance from the American Association for the Study of Liver Diseases. Hepatology 2018;67(1):328-357. doi:10.1002/ hep.29367.

[2] Li Z, Xue J, Chen P, Chen L, Yan S, Liu L. Prevalence of nonalcoholic fatty liver disease in mainland of China: a meta-analysis of published studies. J Gastroenterol Hepatol 2014;29(1):42-51. doi:10.1111/ jgh.12428.

[3] Uygun A. Is that possible to stop or cease the NASH to turn into HCC? J Gastrointest Cancer 2017;48(3):250-255. doi:10.1007/s12029-0179961-6.

[4] Byrne CD, Targher G. NAFLD: a multisystem disease. J Hepatol 2015;62(1):S47-S64. doi:10.1016/j.jhep.2014.12.012.

[5] Fan JG, Kim SU, Wong VW. New trends on obesity and NAFLD in Asia. J Hepatol 2017;67(4):862-873. doi:10.1016/j.jhep.2017.06.003.

[6] Sharpton SR, Ajmera V, Loomba R. Emerging role of the gut microbiome in nonalcoholic fatty liver disease: from composition to function. Clin Gastroenterol Hepatol 2018;17(2):296-306. doi:10.1016/j. cgh.2018.08.065.

[7] Ma J, Zhou Q, Li H. Gut microbiota and nonalcoholic fatty liver disease: insights on mechanisms and therapy. Nutrients 2017;9(10):1124. doi:10.3390/nu9101124.

[8] Hagymasi K, Bacsardi A, Egresi A, Berta E, Tulassay Z, Lengyel G. The role of gut microbiota in chronic liver diseases, and treatment possibilities. Orv Hetil 2018;159(36):1465-1474. doi:10.1556/650.2018.31178.

[9] Lau LHS, Wong SH. Microbiota, obesity and NAFLD. In Yu J. (ed.), Adv Exp Med Biol. Singapore, Springer, 2018:111-125. doi:10.1007/978981-10-8684-7_9.

[10] Behrouz V, Jazayeri S, Aryaeian N, Zahedi MJ, Hosseini F. Effects of probiotic and prebiotic supplementation on leptin, adiponectin, and glycemic parameters in nonalcoholic fatty liver disease: a randomized clinical trial. Middle East J Dig Dis 2017;9(3):150-157. doi:10.15171/ mejdd.2017.66.

[11] Eslamparast T, Eghtesad S, Hekmatdoost A, Poustchi H. Probiotics and nonalcoholic fatty liver disease. Middle East J Dig Dis 2013;5(3):129136.

[12] Lirussi F, Mastropasqua E, Orando S, Orlando R. Probiotics for nonalcoholic fatty liver disease and/or steatohepatitis. Cochrane Database Syst Rev 2007;(1):Cd005165. doi:10.1002/14651858.CD005165. pub2.

[13] Vaughn BP, Rank KM, Khoruts A. Fecal microbiota transplantation: current status in treatment of $\mathrm{Gl}$ and liver disease. Clin Gastroenterol Hepatol 2018;17(2):353-361. doi:10.1016/j.cgh.2018.07.026.
[14] DeFilipp Z, Hohmann E, Jenq RR, Chen YB. Fecal microbiota transplantation: restoring the injured microbiome after allogeneic hematopoietic cell transplantation. Biol Blood Marrow Transplant 2018;25(1):e17-e22. doi:10.1016/j.bbmt.2018.10.022.

[15] Imdad A, Nicholson MR, Tanner-Smith EE, Zackular JP, GomezDuarte OG, Beaulieu DB, et al. Fecal transplantation for treatment of inflammatory bowel disease. Cochrane Database Syst Rev 2018;(11):Cd012774. doi:10.1002/14651858.CD012774.pub2.

[16] de Groot PF, Frissen MN, de Clercq NC, Nieuwdorp M. Fecal microbiota transplantation in metabolic syndrome: History, present and future. Gut Microbes 2017;8(3):253-267. doi:10.1080/19490976.20 17.1293224.

[17] Xu MQ, Cao HL, Wang WQ, Wang S, Cao XC, Yan F, et al. Fecal microbiota transplantation broadening its application beyond intestinal disorders. World J Gastroenterology 2015;21(1):102-111. doi:10.3748/wjg.v21.i1.102.

[18] Zhang F, Cui B, He X, Nie Y, Wu K, Fan D. Microbiota transplantation: concept, methodology and strategy for its modernization. Protein Cell 2018;9(5):462-473. doi:10.1007/s13238-018-0541-8.

[19] Cui B, Li P, Xu L, Peng Z, Xiang J, He Z, et al. Step-up fecal microbiota transplantation (FMT) strategy. Gut Microbes 2016;7(4):323-328. do i: 10.1080/19490976.2016.1151608.

[20] Surawicz CM, Brandt LJ, Binion DG, Ananthakrishnan AN, Curry $\mathrm{SR}$, Gilligan $\mathrm{PH}$, et al. Guidelines for diagnosis, treatment, and prevention of clostridium difficile infections. Am J Gastroenterol 2013;108(4):478-498. doi:10.1038/ajg.2013.4.

[21] de Faria Ghetti F, Oliveira DG, de Oliveira JM, de Castro Ferreira L, Cesar DE, Moreira APB. Influence of gut microbiota on the development and progression of nonalcoholic steatohepatitis. Eur J Nutr 2018;57(3):861-876. doi:10.1007/s00394-017-1524-x.

[22] Ye JZ, Li YT, Wu WR, Shi D, Fang DQ, Yang LY, et al. Dynamic alterations in the gut microbiota and metabolome during the development of methionine-choline-deficient diet-induced nonalcoholic steatohepatitis. World J Gastroenterol 2018;24(23):2468-2481. doi:10.3748/ wjg.v24.i23.2468.

[23] Poeta M, Pierri L, Vajro P. Gut-liver axis derangement in nonalcoholic fatty liver disease. Children (Basel) 2017;4(8):66. doi:10.3390/children4080066.

[24] Tripathi A, Debelius J, Brenner DA, Karin M, Loomba R, Schnabl B, et al. The gut-liver axis and the intersection with the microbiome. Nat Rev Gastroenterol Hepatol 2018;15(7):397-411. doi:10.1038/ s41575-018-0011-z.

[25] Le Roy T, Llopis M, Lepage P, Bruneau A, Rabot S, Bevilacqua C, et al. Intestinal microbiota determines development of nonalcoholic fatty liver disease in mice. Gut 2013;62(12):1787-1794. doi:10.1136/ gutjnl-2012-303816.

[26] Zhou D, Pan Q, Shen F, Cao HX, Ding WJ, Chen YW, et al. Total fecal microbiota transplantation alleviates high-fat diet-induced steatohepatitis in mice via beneficial regulation of gut microbiota. Sci Rep 2017;7(1):1529. doi:10.1038/s41598-017-01751-y.

[27] Soderborg TK, Clark SE, Mulligan CE, Janssen RC, Babcock L, Ir D, et al. The gut microbiota in infants of obese mothers increases inflammation and susceptibility to NAFLD. Nat Commun 2018;9(1):4462. doi:10.1038/s41467-018-06929-0.

[28] Garcia-Lezana T, Raurell I, Bravo M, Torres-Arauz M, Salcedo MT, Santiago $A$, et al. Restoration of a healthy intestinal microbiota normalizes portal hypertension in a rat model of nonalcoholic steatohepatitis. Hepatology 2018;67(4):1485-1498. doi:10.1002/hep.29646.

[29] Deng H, Wang CL, Lai J, Yu SL, Xie DY, Gao ZL. Noninvasive diagnosis of hepatic steatosis using fat attenuation parameter measured by fibrotouch and a new algorithm in CHB patients. Hepat Mon 2016;16(9):e40263. doi:10.5812/hepatmon.40263.

[30] Smits LP, Bouter KE, de Vos WM, Borody TJ, Nieuwdorp M. Therapeutic potential of fecal microbiota transplantation. Gastroenterology 2013;145(5):946-953. doi:10.1053/j.gastro.2013.08.058.

[31] Lynch SV, Pedersen O. The human intestinal microbiome in health and disease. N Engl J Med 2016;375(24):2369-2379. doi:10.1056/ NEJMra1600266.

[32] Alisi A, Carsetti R, Nobili V. Pathogen- or damage-associated molecular patterns during nonalcoholic fatty liver disease development. Hepatology 2011;54(5):1500-1502. doi:10.1002/hep.24611. 
[33] Berni Canani R, Sangwan N, Stefka AT, Nocerino R, Paparo L, Aitoro $\mathrm{R}$, et al. Lactobacillus rhamnosus $\mathrm{GG}$-supplemented formula expands butyrate-producing bacterial strains in food allergic infants. ISME J 2016;10(3):742-750. doi:10.1038/ismej.2015.151.

[34] Guilloteau P, Martin L, Eeckhaut V, Ducatelle R, Zabielski R, Van Immerseel F. From the gut to the peripheral tissues: the multiple effects of butyrate. Nutr Res Rev 2010;23(02):366-384. doi:10.1017/ s0954422410000247.

[35] Jin CJ, Sellmann C, Engstler AJ, Ziegenhardt D, Bergheim I. Supplementation of sodium butyrate protects mice from the development of nonalcoholic steatohepatitis (NASH). Br J Nutr 2015;114(11):17451755. doi:10.1017/s0007114515003621.

[36] Smith PM, Howitt MR, Panikov N, Michaud M, Gallini CA, Bohlooly YM, et al. The microbial metabolites, short-chain fatty acids, regulate colonic Treg cell homeostasis. Science 2013;341(6145):569-573. doi:10.1126/science.1241165.

[37] Park JS, Lee EJ, Lee JC, Kim WK, Kim HS. Anti-inflammatory effects of short chain fatty acids in IFN-gamma-stimulated RAW 264.7 murine macrophage cells: involvement of NF-kappaB and ERK signaling pathways. Int Immunopharmacol 2007;7(1):70-77. doi:10.1016/j. intimp.2006.08.015.

[38] Zhang Q, Wu Y, Wang J, Wu G, Long W, Xue Z, et al. Accelerated dysbiosis of gut microbiota during aggravation of DSS-induced colitis by a butyrate-producing bacterium. Sci Rep 2016;6:27572. doi:10.1038/ srep27572.

[39] Ploger S, Stumpff F, Penner GB, Schulzke JD, Gabel G, Martens H, et al. Microbial butyrate and its role for barrier function in the gastrointestinal tract. Ann N Y Acad Sci 2012;1258(1):52-59. doi:10.1111/ j.1749-6632.2012.06553.x.

[40] Tan J, McKenzie C, Potamitis M, Thorburn AN, Mackay CR, Macia L. The role of short-chain fatty acids in health and disease. Adv Immunol 2014;121:91-119. doi:10.1016/b978-0-12-800100-4.00003-9.

[41] Gan LT, Van Rooyen DM, Koina ME, McCuskey RS, Teoh NC, Farrell GC. Hepatocyte free cholesterol lipotoxicity results from JNK1-mediated mitochondrial injury and is HMGB1 and TLR4-dependent. J Hepatol 2014;61(6):1376-1384. doi:10.1016/j.jhep.2014.07.024.

[42] Kubes P, Mehal WZ. Sterile inflammation in the liver. Gastroenterology 2012;143(5):1158-1172. doi:10.1053/j.gastro.2012.09.008. 